\title{
Effectiveness of treadmill training on gait function in children with cerebral palsy: meta-analysis
}

\author{
Yong-Gu Han, Chang-Kyo Yun* \\ Department of Physical Therapy, College of Rehabilitation Sciences, Daegu University, Gyeongsan, Korea
}

The purpose of this review was to analysis the effects of treadmill training on gait function in children with cerebral palsy. Cumulative Index to Nursing and Allied Health Literature (CINAHL), Cochrane Central Register of Controlled Trials, Physiotherapy Evidence Database (PEDro), PubMed and Web of Science were searched. Investigating the effects of treadmill training on gait endurance, gait speed, limb support time, cadence, and step length in children with cerebral palsy. Similar outcomes were pooled by calculating the standardized mean difference. Of the eight studies, 179 participants were included. The average PEDro score was $6.25 / 10$. The results of the sensitivity test for bias evaluation using the duval and tweedie's trim and fill method showed low publication bias. The test regarding the effect of treadmill training on overall gait function yielded a moderate effect size of 0.53 , which was a statistically significant effect as its confidence interval did not include. The overall effect size of gait endurance was 0.85 . The overall effect size of gait speed and limb support time were 0.52 and 0.73 . The overall effect size of cadence and step length were 0.14 and 0.21 , indicating a nonsignificant improvement. These findings suggested that treadmill training on cerebral palsy was effective for gait endurance, gait speed and limb support time than cadence and step length.

Keywords: Cerebral palsy, Gait function, Meta-analysis, Treadmill training

\section{INTRODUCTION}

Most children with cerebral palsy have impaired gait function due to involuntary muscle spasm and muscle weakness (Nicholson et al., 2018). In particular, according to the International Classification of Functioning, Disability and Health model defined by the World Health Organization, the latest intervention goal is focused on not only physical structure but also daily activities and social participation, in which gait function plays crucial roles (Lepage et al., 1998; Schiariti et al., 2015). In fact, children with cerebral palsy develop limitations in gait function with regard to both structure and activity (Hashiguchi et al., 2018). Thus, improving both these aspects is the primary therapeutic goal of treatment among children with cerebral palsy (Nicholson et al., 2018).

In the clinical setting, a wide range of equipment is used as a supplement to conventional therapy in order to improve the gait ability of children with cerebral palsy (Saquetto et al., 2015). The treadmill is one of the equipment that has long been used for rehabilitation of the nervous system (Birgani et al., 2016; Klamroth et al., 2019; Macko et al., 2001). The treadmill effectively improves gait ability through repeated weight loading on an individual's lower limbs (Polese et al., 2013). In particular, the positive effects of repetitive task-oriented training involving the treadmill on motor learning among subjects have been reported by multiple systematic reviews (Mehrholz et al., 2017; Polese et al., 2013; Willoughby et al., 2009).

The latest trend in evidence-based research highlights the importance of qualitative as well as quantitative research (Schalock et al., 2017). In particular, a systematic review with a meta-analytic technique can be used to make an inference on the combined results of previously published individual studies, using a systematic method (Borenstein et al., 2011). With the increasing need for qualitative research in the field, studies on the effects of treadmills on various types of patients have been conducted, and they have
${ }^{*}$ Corresponding author: Chang-Kyo Yun (D) https://orcid.org/0000-0003-2094-4239 Department of Physical Therapy, College of Rehabilitation Sciences, Daegu

University, 201 Daegudae-ro, Jillyang-eup, Gyeongsan 38453, Korea

E-mail: puhaha1116@naver.com

Received: December 23, 2019 / Accepted: January 30, 2020
This is an Open Access article distributed under the terms of the Creative Commons Attribution Non-Commercial License (https://creativecommons.org/licenses/by-nc/4.0/) which permits unrestricted non-commercial use, distribution, and reproduction in any medium, provided the original work is properly cited. 
reported positive effects (Mehrholz et al., 2017; Polese et al., 2013; Zwicker et al., 2010).

However, qualitative research on the effects of treadmill training in children with cerebral palsy remains scarce. Such research requires analysis of multiple aspects of gait function, as interaction of multiple joints is involved. However, previous studies on children with cerebral palsy have many areas that need to be improved (Schwartz and Rozumalski, 2008). The systematic review by Zwicker et al. (2010) did not employ the meta-analytic technique, and thus, it offers no effect size estimated quantitatively. The study by Willoughby. (2009) examined only two aspects of gait function, namely gait speed and endurance, neglecting other important aspects. As gait function can be measured in various ways and measures are continuously being developed, research needs to incorporate instruments that measure various aspects of gait function. Other systematic reviews and meta-analytic studies had limitations in generalizing their findings because the subjects were children with developmental disorders, including children with Down syndrome and those with cerebral palsy (Johnson, 2009). This indicates the need for a systematic review and meta-analysis on the effects of the treadmill in children with cerebral palsy, improving on previous studies. The purpose of this study was to investigate the effects of the treadmill on specific aspects of gait function (gait endurance, gait speed, limb support time, cadence, and step length) in children with cerebral palsy through a systematic review and meta-analysis.

\section{MATERIALS AND METHODS}

\section{Data sources and searches}

This review conducted by Preferred Reporting Items for Systematic Reviews and Meta-Analyses (PRISMA) statement (Moher et al., 2015). Two authors independently conducted searches in August 2019 using electronic data base: Cumulative Index to Nursing and Allied Health Literature (CINAHL), Cochrane Central Register of Controlled Trials, Physiotherapy Evidence Database (PEDro), PubMed, and Web of Science. Keywords or Mesh terms (if applicable) used for the search include the following: spastic diplegia(s) or spastic hemiplegia(s) or cerebral palsy(s) or cerebral palsies or little disease or infantile palsies or spastic diplegic or spastic hemiplegic or spastic quadriplegia(s) or spastic, treadmill training or partial body weight treadmill or body weight supported training or treadmill therapy or partial body weight treadmill, gait or walking or walking ability or walking abilities or locomotor training or locomotion or gait function or mobility (Fig. 1).

\section{Data processing}

This systematic review and meta-analysis considered studies that met the following inclusion criteria: (a) enrolled children aged 6-18 years diagnosed with cerebral palsy, (b) compared treadmill training with conventional therapy or a control, (c) measured the outcome with regard to gait speed, limb support time, cadence, and/or step length, and (d) written in English. The ex-

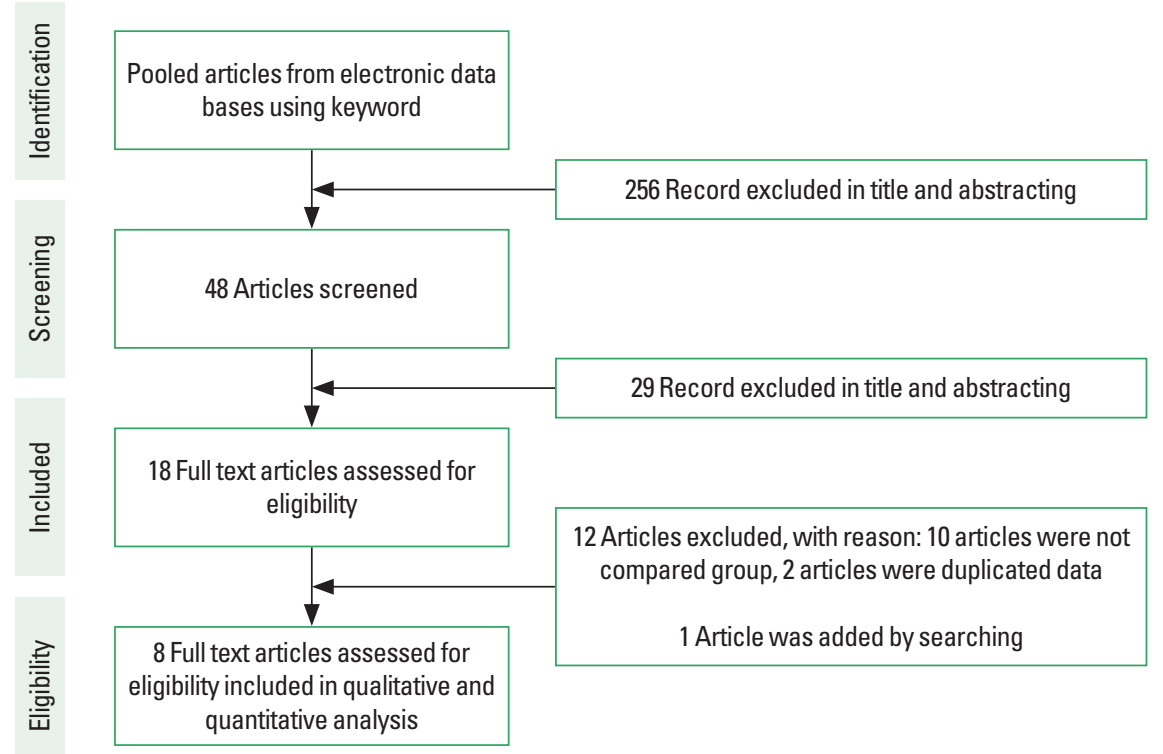

Fig. 1. Eligibility and data-synthesis PRISMA (Preferred Reporting Items for Systematic Reviews and Meta-Analyses) flow diagram. 
clusion criteria were as follows: (a) insufficient data for estimation of the effect size and (b) demonstration of an immediate effect. Data were coded by individuals skilled in meta-analysis, and the coding was completed after all authors of this study reached an agreement. The author names, published year, study participants, cerebral palsy type, the gross motor function classification scale level, dosage, assessment tools were recorded for data coding.

\section{Data extraction and quality assessment}

In this study, data were organized using the Patient Intervention Comparator Outcome framework as follows: participants were children diagnosed with cerebral palsy; intervention was treadmill training; comparator was conventional therapy, nontherapy, or other therapy; and outcome was the effect of treadmill training on gait function in children with cerebral palsy. The assessment of gait function included gait speed, gait endurance, limb support time, cadence, and step length. The quality of the included studies was evaluated using the PEDro scale (Yamato et al., 2018). The scale has 11 items (eligibility criteria, random allocation, concealed allocation, baseline comparability, blinded subjects, blinded therapists, blinded assessors, adequate follow-up, intention to treat, between-group, point estimators), and the total score is interpreted as follows: 9-10: excellent; 6-8: good; 4-6: fair; and <4: poor. The publication bias of the included studies was assessed using the duval and tweedie's trim and fill method (Macaskill et al., 2001). Since this study was a meta-analysis of published articles, this study was exempt from Institutional Review Board review.

\section{Data synthesis and analysis}

Data analysis was performed using Comprehensive meta-analysis software. According to Cohen criteria, the effect size is considered small when $\leq 0.3$, moderate when $0.4-0.7$, and large when $\geq 0.8$ (Borenstein et al., 2011).

\section{RESULTS}

\section{Characteristics of included trials}

With regard to the characteristics of the included studies (Table 1) (Cherng et al., 2007; Chrysagis et al., 2012; Dodd and Foley, 2007; Gharib et al., 2011; Grecco et al., 2013; Hösl et al., 2018; Johnston et al., 2011; Willoughby et al., 2010), Eight studies were considered, and the study by Cherng was divided into two studies according to the switch between the experimental and control groups because of the counterbalance design.

Table 1. Study characteristics

\begin{tabular}{|c|c|c|c|c|c|c|c|}
\hline Study & Group & $\begin{array}{l}\text { No. of participants } \\
\text { Intervention }\end{array}$ & $\begin{array}{c}\text { Sex } \\
\text { (male/female) }\end{array}$ & $\begin{array}{c}\text { Age (yr), } \\
\text { mean } \pm \text { SD }\end{array}$ & $\begin{array}{l}\text { Cerebral palsy type } \\
\text { GMFCS }\end{array}$ & Dosage & Outcome \\
\hline \multirow[t]{2}{*}{$\begin{array}{l}\text { Dodd and Foley } \\
\text { (2007) }\end{array}$} & Experimental & $\begin{array}{l}7 \\
\text { PBWSTT+usual physical } \\
\text { activity program }\end{array}$ & $5 / 2$ & $8.5 \pm 2.6$ & $\begin{array}{l}\text { Athetoid quadriplegia } 4 \\
\text { Spastic diplegia } 1 \\
\text { Spastic quadriplegia } 2 \\
\text { GMFCS level 3(2), 4(5) }\end{array}$ & $30 \min 2$ day $\times 6 \mathrm{wk}$ & \multirow[t]{2}{*}{$\begin{array}{l}10 \mathrm{MWT} \\
10-\mathrm{MWT}\end{array}$} \\
\hline & Control & $\begin{array}{l}\text { U } \\
\text { Usual physical activity } \\
\text { program }\end{array}$ & $5 / 2$ & $9.5 \pm 2.9$ & $\begin{array}{l}\text { Athetoid quadriplegia } 4 \\
\text { Spastic diplegia } 1 \\
\text { Spastic quadriplegia } 2 \\
\text { GMFCS level } 3(2), 4(5)\end{array}$ & & \\
\hline \multirow[t]{2}{*}{$\begin{array}{l}\text { Cherng et al. } \\
\text { (2007) }\end{array}$} & Experimental & $\begin{array}{l}4 \\
\text { TBWS+regular therapeutic } \\
\text { treatment }\end{array}$ & $2 / 2$ & $4.5 \pm 2$ & $\begin{array}{l}\text { Spastic diplegia } 4 \\
\text { GMFCS level 2(1), 3(3) }\end{array}$ & \multirow[t]{2}{*}{$\begin{array}{l}20 \min 2 \text { or } 3 \text { day } x \\
12 \text { wk }\end{array}$} & \multirow{2}{*}{$\begin{array}{l}\text { Gait speed } \\
\text { Cadence } \\
\text { Gait stride length } \\
\text { Gait double } \\
\text { limb support }\end{array}$} \\
\hline & Control & $\begin{array}{c}4 \\
\text { Regular therapeutic treatment }\end{array}$ & $4 / 0$ & $4.5 \pm 2$ & $\begin{array}{l}\text { Spastic diplegia } 4 \\
\text { GMFCS level 2(1), 3(3) }\end{array}$ & & \\
\hline \multirow[t]{2}{*}{$\begin{array}{l}\text { Willoughby et al. } \\
\text { (2010) }\end{array}$} & Experimental & $\begin{array}{c}12 \\
\text { PBWSTT+usual physiotherapy }\end{array}$ & $6 / 6$ & $10.3 \pm 3.1$ & $\begin{array}{l}\text { Not report } \\
\text { GMFCS level 3(5), 4(7) }\end{array}$ & \multirow[t]{2}{*}{30 min 2 day $\times 9$ wk } & \multirow{2}{*}{$\begin{array}{l}10 \mathrm{MWT} \\
10-\mathrm{MWT} \\
\text { SFA }\end{array}$} \\
\hline & Control & $\begin{array}{l}14 \\
\text { Over ground walking+usual } \\
\text { physiotherapy }\end{array}$ & $9 / 5$ & $11.2 \pm 4.1$ & $\begin{array}{l}\text { Not report } \\
\text { GMFCS level 3(3), 4(11) }\end{array}$ & & \\
\hline \multirow[t]{2}{*}{$\begin{array}{l}\text { Gharib et al. } \\
\text { (2011) }\end{array}$} & Experimental & $\begin{array}{c}15 \\
\text { TGT+traditional physical therapy }\end{array}$ & $10 / 5$ & $11.9 \pm 1.1$ & $\begin{array}{l}\text { Spastic hemiplegia } 15 \\
\text { GMFCS level 2(15) }\end{array}$ & \multirow[t]{2}{*}{$15 \min 3$ day $\times 12$ wk } & \multirow{2}{*}{$\begin{array}{l}\text { Gait speed } \\
\text { Step length } \\
\text { Ambulation index } \\
\text { Time of support }\end{array}$} \\
\hline & Control & $\begin{array}{c}15 \\
\text { Traditional physical therapy }\end{array}$ & $6 / 9$ & $11.2 \pm 1.1$ & $\begin{array}{l}\text { Spastic hemiplegia } 15 \\
\text { GMFCS level 2(15) }\end{array}$ & & \\
\hline
\end{tabular}

(Continued to the next page) 
Table 1. Continued

\begin{tabular}{|c|c|c|c|c|c|c|c|}
\hline Study & Group & $\begin{array}{l}\text { No. of participants } \\
\text { Intervention }\end{array}$ & $\begin{array}{c}\text { Sex } \\
\text { (male/female) }\end{array}$ & $\begin{array}{c}\text { Age (yr), } \\
\text { mean } \pm \text { SD }\end{array}$ & $\begin{array}{c}\text { Cerebral palsy type } \\
\text { GMFCS }\end{array}$ & Dosage & Outcome \\
\hline \multirow[t]{2}{*}{$\begin{array}{l}\text { Johnston et al. } \\
\text { (2011) }\end{array}$} & Experimental & SSTTEP & $7 / 7$ & $9.7 \pm 2.2$ & $\begin{array}{l}\text { Spastic diplegia } 8 \\
\text { Spastic quadriplegia } 6 \\
\text { GMFCS level 2(1), 3(9), } \\
\quad 4(4)\end{array}$ & 30 min 5 day $\times 12$ wk & \multirow{2}{*}{$\begin{array}{l}\text { Spasticity } \\
\text { Strength } \\
\text { Motor control } \\
\text { GMFM } \\
\text { Cadence } \\
\text { Step length } \\
\text { PODCl }\end{array}$} \\
\hline & Control & Strengthening & $7 / 5$ & $9.6 \pm 2.4$ & $\begin{array}{l}\text { Spastic diplegia } 4 \\
\text { Spastic quadriplegia } 6 \\
\text { Spastic triplegia } 2 \\
\text { GMFCS level 2(1), 3(6), } \\
5(5)\end{array}$ & & \\
\hline \multirow[t]{2}{*}{$\begin{array}{l}\text { Chrysagis et al. } \\
\text { (2012) }\end{array}$} & Experimental & TGT & $6 / 5$ & $15.9 \pm 2$ & $\begin{array}{l}\text { Spastic diplegia } 9 \\
\text { Spastic tetraplegia } 2 \\
\text { GMFCS level 1(3), 2(4), } \\
\quad 3(4)\end{array}$ & 30 min 3 day $\times 12$ wk & \multirow[t]{2}{*}{$\begin{array}{l}\text { GMFM } \\
\text { 10-MWT } \\
\text { MAS }\end{array}$} \\
\hline & Control & $\begin{array}{c}11 \\
\text { Conventional physiotherapy }\end{array}$ & $7 / 4$ & $16 \pm 1.6$ & $\begin{array}{l}\text { Spastic diplegia } 10 \\
\text { Spastic tetraplegia } 1 \\
\text { GMFCS level 1(2), 2(5), } \\
3(4)\end{array}$ & & \\
\hline \multirow[t]{2}{*}{$\begin{array}{l}\text { Grecco et al. } \\
(2013)\end{array}$} & Experimental & TGT & $6 / 10$ & $6.8 \pm 2.6$ & $\begin{array}{l}\text { Not report } \\
\text { GMFCS level 1(5), 2(8), } \\
3(3)\end{array}$ & $30 \min 2$ day $\times 7 w k$ & \multirow[t]{2}{*}{$\begin{array}{l}6 \mathrm{MWT} \\
\text { TUG } \\
\text { BBS }\end{array}$} \\
\hline & Control & $\begin{array}{c}17 \\
\text { Over ground walking }\end{array}$ & $9 / 8$ & $6.0 \pm 1.5$ & $\begin{array}{l}\text { Not report } \\
\text { GMFCS level 1(8), 2(7), } \\
\text { 3(2) }\end{array}$ & & \\
\hline \multirow[t]{2}{*}{$\begin{array}{l}\text { Hösl et al. } \\
\text { (2018) }\end{array}$} & Experimental & $\mathrm{BDT}$ & Not report & $12 \pm 4$ & $\begin{array}{l}\text { Spastic diplegia } 8 \\
\text { Spastic hemiplegia } 2 \\
\text { GMFCS level 1(4), 2(6) }\end{array}$ & 23 min 3 day $\times 9$ wk & \multirow{2}{*}{$\begin{array}{l}\text { Gait speed } \\
\text { Cadence } \\
\text { Step length } \\
\text { GMFM } \\
\text { TUG } \\
\text { MAS } \\
\text { Ankle joint } \\
\text { Muscle-tendon } \\
\text { Total strain } \\
\text { Stiffness }\end{array}$} \\
\hline & Control & Stretching 10 & Not report & $12 \pm 4$ & $\begin{array}{l}\text { Spastic diplegia } 8 \\
\text { Spastic hemiplegia } 2 \\
\text { GMFCS level 1(4), 2(6) }\end{array}$ & & \\
\hline
\end{tabular}

SD, standard deviation; GMFCS, gross motor function classification scale; PBWST, partial body weight supported treadmill training; 10MWT, 10-min walking test; 10-MWT, 10-m walking test; TBWS, treadmill training with body weight support; SSTTEP, supported speed treadmill training exercise program; GMFM, gross motor function measure; PODCl, pediatric outcomes data collection instrument; TGT, treadmill gait training; $6 \mathrm{MWT}$, 6-min walking test; BBS, Berg balance scale; PBWST, partial body weight supported treadmill training; SFA, school function assessment travel scale; TUG, Timed Up and Go test; MAS, modified Ashworth scale; BDT, backward downhill treadmill training.

\section{Homogeneity test for selecting model}

As the results of the homogeneity test $(\mathrm{Q}=21.41, P=0.004$, $\left.I^{2}=64.95\right)$ and visual inspection indicated a high level of homogeneity among the eight included studies, we used the random-effect model to derive estimates. In addition, in subgroup analyses, the estimates for gait speed were obtained using the random-effect model as a high level of heterogeneity was suggested, whereas the estimates for gait endurance, limb support time, cadence, and step length were obtained using the fixed-effect model as a low level of heterogeneity was suggested.

\section{Quality assessment}

On the PEDro scale, the mean score for the included studies was 6.25. Individual item scores are shown in (Table 2).

\section{Publication bias assessment}

The results of the sensitivity test for bias evaluation using the duval and tweedie's trim and fill method showed that the correlation values and observed values were the same, suggesting low publication bias (Table 3).

\section{Combined effect of treadmill training}

The test regarding the effect of treadmill training on overall 
Table 2. Quality assessment (PEDro scale)

\begin{tabular}{|c|c|c|c|c|c|c|c|c|c|c|c|c|}
\hline \multirow{2}{*}{ Study } & \multicolumn{11}{|c|}{ Item } & \multirow{2}{*}{ Tota } \\
\hline & 1 & 2 & 3 & 4 & 5 & 6 & 7 & 8 & 9 & 10 & 11 & \\
\hline Dodd and Foley (2007) & $\sqrt{ }$ & 1 & 1 & 1 & 1 & 0 & 0 & 1 & 1 & 1 & 1 & 8 \\
\hline Cherng et al. (2007) & $\sqrt{ }$ & 0 & 1 & 0 & 1 & 0 & 0 & 1 & 1 & 0 & 1 & 5 \\
\hline Willoughby et al. (2010) & $\sqrt{ }$ & 1 & 1 & 1 & 0 & 0 & 0 & 0 & 1 & 1 & 1 & 6 \\
\hline Gharib et al. (2011) & $\sqrt{ }$ & 1 & 1 & 1 & 1 & 0 & 0 & 1 & 1 & 1 & 1 & 8 \\
\hline Johnston et al. (2011) & $\sqrt{ }$ & 1 & 1 & 0 & 1 & 0 & 0 & 0 & 0 & 0 & 1 & 4 \\
\hline Chrysagis et al. (2012) & $\sqrt{ }$ & 1 & 1 & 1 & 1 & 0 & 0 & 1 & 1 & 1 & 1 & 8 \\
\hline Grecco et al. (2013) & $\sqrt{ }$ & 0 & 1 & 1 & 1 & 0 & 0 & 1 & 1 & 1 & 1 & 7 \\
\hline Hösl et al. (2018) & $\sqrt{ }$ & 1 & 0 & 1 & 0 & 0 & 0 & 0 & 0 & 1 & 1 & 4 \\
\hline
\end{tabular}

PEDro, physiotherapy evidence database; 1, eligibility criteria; 2, random allocation; 3, concealed allocation; 4, baseline comparability; 5, blinded subjects; 6, blinded therapists; 7, blinded assessors; 8 , adequate follow-up; 9 , intention to treat; 10, between group; 11, point estimators.

\begin{tabular}{|c|c|c|c|c|c|c|c|c|}
\hline \multirow[t]{2}{*}{$\underline{\text { Study name }}$} & \multicolumn{2}{|c|}{ Subgroup within study } & \multicolumn{4}{|c|}{ Statistics for each study } & \multirow{2}{*}{\multicolumn{2}{|c|}{ Z-Value p-Value }} \\
\hline & & $\begin{array}{c}\text { Std diff } \\
\text { in means }\end{array}$ & $\begin{array}{c}\text { Standard } \\
\text { error }\end{array}$ & Variance & $\begin{array}{l}\text { Lower } \\
\text { limit }\end{array}$ & $\begin{array}{c}\text { Upper } \\
\text { limit }\end{array}$ & & \\
\hline Cheong et al 2007 & Combined & 0.083 & 0.408 & 0.167 & -0.717 & 0.884 & 0.204 & 0.838 \\
\hline Cheong et al 2007 & Combined & 0.416 & 0.415 & 0.172 & -0.397 & 1.229 & 1.003 & 0.316 \\
\hline Chrysagis et al 2012 & Gait speed & 1.016 & 0.453 & 0.205 & 0.128 & 1.904 & 2.243 & 0.025 \\
\hline Dodd et al 2007 & Combined & 0.561 & 0.385 & 0.148 & -0.194 & 1.317 & 1.457 & 0.145 \\
\hline Gharib et al 2011 & Combined & 0.535 & 0.215 & 0.046 & 0.113 & 0.957 & 2.482 & 0.013 \\
\hline Grecco et al 2013 & Combined & 1.603 & 0.283 & 0.080 & 1.048 & 2.159 & 5.656 & 0.000 \\
\hline Hosl et al 2018 & Combined & 0.217 & 0.224 & 0.050 & -0.223 & 0.657 & 0.966 & 0.334 \\
\hline Johnston et al 2011 & Combined & 0.113 & 0.227 & 0.052 & -0.333 & 0.558 & 0.496 & 0.620 \\
\hline \multirow{2}{*}{\multicolumn{2}{|c|}{ Willoughby et al 2010 Combined }} & 0.253 & 0.280 & 0.078 & -0.295 & 0.801 & 0.906 & 0.365 \\
\hline & & & 0.169 & 0.029 & 77 & 0849 & 87 & \\
\hline
\end{tabular}

Fig. 2. Combined effect size of treadmill training. $\mathrm{Cl}$, confidence interval.

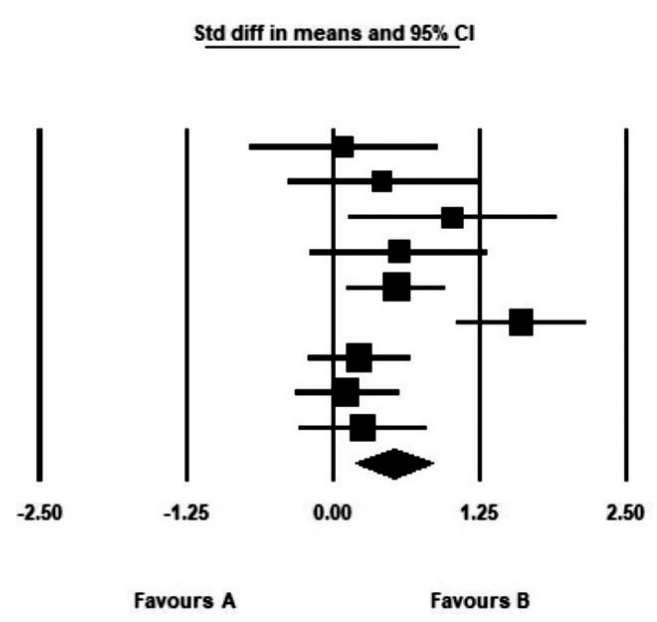

Table 3. Publication bias

\begin{tabular}{lcccc}
\hline Value & Study & Point estimate & $95 \% \mathrm{Cl}$ & 0-value \\
\hline Observed & - & 0.52 & $0.19-0.85$ & 22.82 \\
Adjusted & 0 & 0.52 & $0.19-0.85$ & 22.82 \\
\hline
\end{tabular}

$\mathrm{Cl}$, confidence interval.

gait function yielded a moderate effect size of 0.52 (95\% confidence interval [CI], 0.19-0.85), which was a statistically significant effect as its confidence interval did not include (Fig. 2).

\section{Gait endurance}

There were three studies that included outcome variables that measured gait endurance. The overall effect size was 0.85 (95\% CI, 0.37-1.34), indicating a large effect size (Fig. 3).

\section{Gait speed}

There were nine studies that included outcome variables that measured gait speed. The overall effect size was 0.53 (95\% CI, 0.15-0.92), indicating a medium effect size (Fig. 4).

\section{Limb support time}

There were three studies that included outcome variables that measured limb support time. The overall effect size was 0.73 (95\% CI, 0.14-1.34), indicating a moderate effect size (Fig. 5).

\section{Cadence}

There were four studies that included outcome variables that measured cadence. The overall effect size was 0.14 (95\% CI, -0.36 to 0.64 ), indicating a nonsignificant improvement (Fig. 6).

\section{Step length}

There were three studies that included outcome variables that measured step length. The overall effect size was 0.21 (95\% CI, -0.24 to 0.67 ), indicating a non-significant improvement (Fig. 7). 


\begin{tabular}{|c|c|c|c|c|c|c|c|c|}
\hline Study name & Subgroup withi & idy & & tatistics fo & or each & study & & \\
\hline & & $\begin{array}{c}\text { Std diff } \\
\text { in means }\end{array}$ & $\begin{array}{c}\text { Standard } \\
\text { error }\end{array}$ & Variance & $\begin{array}{c}\text { Lower } \\
\text { limit }\end{array}$ & $\begin{array}{l}\text { Upper } \\
\text { limit }\end{array}$ & Z-Value & o-Val \\
\hline Dodd et al 2007 & Gait endurance & 0.576 & 0.545 & 0.298 & -0.494 & 1.645 & 1.055 & 0.291 \\
\hline Grecco et al 2013 & Gat endurance & 1.444 & 0.391 & 0.153 & 0.677 & 2.210 & 3.692 & 0.00 \\
\hline Willoughby et al 20 & 0 Gait endurance & 0.388 & 0.397 & 0.158 & -0.391 & 1.166 & 0.976 & 0.3 \\
\hline & & 0.852 & 0.248 & 0.062 & 0.365 & 1.338 & 3.433 & 0.0 \\
\hline
\end{tabular}

Fig. 3. Effect size at gait endurance. Cl, confidence interval.

\begin{tabular}{|c|c|c|c|c|c|c|c|c|}
\hline \multirow[t]{2}{*}{ Study name } & \multicolumn{2}{|c|}{ Subgroup within study } & \multicolumn{4}{|c|}{ Statistics for each study } & \multirow{2}{*}{\multicolumn{2}{|c|}{ Z-Value p-Value }} \\
\hline & & $\begin{array}{l}\text { Std diff } \\
\text { in means }\end{array}$ & $\begin{array}{c}\text { Standard } \\
\text { error }\end{array}$ & Variance & Lower & $\begin{array}{c}\text { Upper } \\
\text { limit }\end{array}$ & & \\
\hline Cheong et al 2007 & Gait speed & 0.075 & 0.707 & 0.500 & -1.311 & 1.461 & 0.106 & 0.915 \\
\hline Cheong et al 2007 & Gait speed & 0.321 & 0.712 & 0.506 & -1.073 & 1.716 & 0.452 & 0.652 \\
\hline Chrysagis et al 2012 & Gait speed & 1.016 & 0.453 & 0.205 & 0.128 & 1.904 & 2.243 & 0.025 \\
\hline Dodd et al 2007 & Gait speed & 0.547 & 0.544 & 0.296 & -0.520 & 1.614 & 1.005 & 0.315 \\
\hline Gharib et al 2011 & Gait speed & 0.420 & 0.369 & 0.136 & -0.303 & 1.144 & 1.139 & 0.255 \\
\hline Grecco et al 2013 & Gait speed & 1.780 & 0.411 & 0.169 & 0.973 & 2.586 & 4.326 & 0.000 \\
\hline Hosl et al 2018 & Combined & 0.192 & 0.317 & 0.101 & -0.430 & 0.814 & 0.605 & 0.545 \\
\hline Johnston et al 2011 & Gait speed & 0.172 & 0.394 & 0.155 & -0.601 & 0.944 & 0.436 & 0.663 \\
\hline \multirow{2}{*}{\multicolumn{2}{|c|}{ Willoughby et al 2010 Gait speed }} & 0.121 & 0.394 & 0.155 & -0.651 & 0.893 & 0.307 & 0.759 \\
\hline & & 0.532 & 0.197 & 0.039 & 0.146 & 0.918 & 2.703 & 0.007 \\
\hline
\end{tabular}

Fig. 4. Effect size at gait speed. Cl, confidence interval.

\begin{tabular}{|c|c|c|c|c|c|c|c|c|}
\hline \multirow[t]{2}{*}{ Study name } & \multicolumn{2}{|l|}{ Subgroup within study } & \multicolumn{4}{|c|}{ Statistics for each study } & \multirow{2}{*}{\multicolumn{2}{|c|}{ Z-Value p-Value }} \\
\hline & & $\begin{array}{l}\text { Std diff } \\
\text { in means }\end{array}$ & $\begin{array}{c}\text { Standard } \\
\text { error }\end{array}$ & Variance & $\begin{array}{l}\text { Lower } \\
\text { limit }\end{array}$ & $\begin{array}{c}\text { Upper } \\
\text { limit }\end{array}$ & & \\
\hline Cheong et al 2007 & Limb support & 0.097 & 0.708 & 0.501 & -1.289 & 1.484 & 0.138 & 0.890 \\
\hline Cheong et al 2007 & Limb support & 0.812 & 0.736 & 0.541 & -0.630 & 2.254 & 1.104 & 0.269 \\
\hline \multirow[t]{2}{*}{ Gharib et al 2011} & Limb support & 0.906 & 0.383 & 0.147 & 0.155 & 1.658 & 2.364 & 0.018 \\
\hline & & 0.738 & 0.306 & 0.094 & 0.138 & 1.339 & 2.409 & 0.016 \\
\hline
\end{tabular}

Fig. 5. Effect size limb support time. Cl, confidence interval.

\begin{tabular}{|c|c|c|c|c|c|c|c|c|}
\hline \multirow[t]{2}{*}{ Study name } & \multicolumn{2}{|l|}{ Subgroup within study } & \multicolumn{4}{|c|}{ Statistics for each study } & \multirow{2}{*}{\multicolumn{2}{|c|}{ Z-Value p-Value }} \\
\hline & & $\begin{array}{l}\text { Std diff } \\
\text { in means }\end{array}$ & $\begin{array}{c}\text { Standard } \\
\text { error }\end{array}$ & Variance & $\begin{array}{l}\text { Lower } \\
\text { limit }\end{array}$ & $\begin{array}{c}\text { Upper } \\
\text { limit }\end{array}$ & & \\
\hline Cheong et al 2007 & Cadence & 0.077 & 0.707 & 0.500 & -1.309 & 1.464 & 0.109 & 0.913 \\
\hline Cheong et al 2007 & Cadence & 0.142 & 0.708 & 0.501 & -1.246 & 1.530 & 0.201 & 0.841 \\
\hline Johnston et al 2011 & Cadence & 0.063 & 0.393 & 0.155 & -0.708 & 0.834 & 0.159 & 0.873 \\
\hline Hosl et al 2018 & Cadence & 0.267 & 0.449 & 0.202 & -0.614 & 1.147 & 0.594 & 0.553 \\
\hline & & 0.141 & 0.255 & 0.065 & -0.359 & 0.640 & 0.552 & 0.581 \\
\hline
\end{tabular}

Fig. 6. Effect size at cadence. Cl, confidence interval.

Std diff in means and $95 \% \mathrm{Cl}$

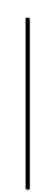

$-1.25$

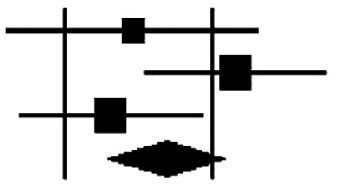

0.00

1.25

2.50
Favours B

\section{Std diff in means and $95 \% \mathrm{Cl}$}

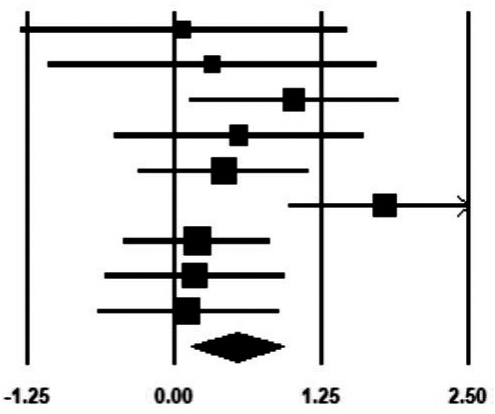

Favours A

Favours B

Std diff in means and $95 \% \mathrm{Cl}$

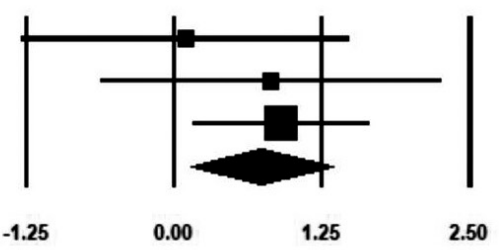

Favours A

Favours B

Std diff in means and $95 \% \mathrm{Cl}$

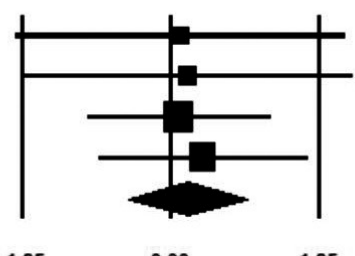

$-1.25$

0.00

1.25

2.50 
Fig. 7. Effect size at step length. Cl, confidence interval.
0.216

0.103

0.213
Study name Subgroup within study

Statistics for each study

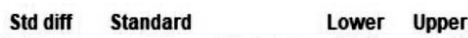

Std diff Standard Lower Upper

$\begin{array}{lllll}0.307 & 0.367 & 0.135 & -0.413 & 1.027\end{array}$

Gharib et al 2011 Step length

Hosl et al $2018 \quad$ Step length

Johnston et al 2011 Step length
0.449

0.394

0.230 $\begin{array}{lll}0.201 & -0.663 & 1.095\end{array}$

$\begin{array}{lll}0.155 & -0.668 & 0.875\end{array}$

$\begin{array}{lll}0.053 & -0.238 & 0.665\end{array}$

$\begin{array}{rr}\text { Z-Value } & \text { p-Value } \\ 0.835 & 0.404 \\ 0.482 & 0.630 \\ 0.263 & 0.793 \\ 0.925 & 0.355\end{array}$

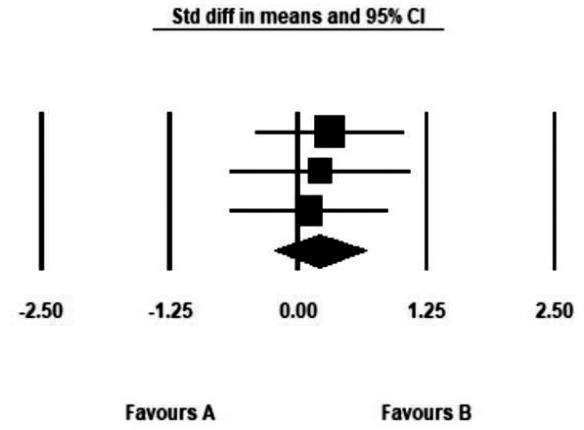

\section{DISCUSSION}

In this review, we investigated the effects of treadmill training on gait function in children with cerebral palsy using meta-analysis. We analyzed eight studies, and the quality assessment yielded a mean score of 6.25 out of 10 . The meta-analysis focused on gait function (gait endurance, gait speed, limb support time, cadence, and step length). Limb support time consists of reduced double limb support and increased one limb support time (affected or more affected side). Most children with cerebral palsy have impaired gait speed, gait endurance and asymmetrical movement pattern during gait cycle (Cherng et al., 2007; Hashiguchi et al., 2018). Gait endurance, gait speed, limb support time, cadence and step length are important clinical goal for the rehabilitation of children with cerebral palsy (Cherng et al., 2007; Chrysagis et al., 2012; Hösl et al., 2018). Therefore, in this review we included five variables to estimate gait function. The analysis of overall gait function yielded a moderate effect size. Subgroup analyses yielded a large effect size for gait endurance and a moderate effect size for gait speed and limb support time, whereas there were no significant differences in cadence and step length between the experimental and comparison groups.

Studies regarding the effects of treadmill training on gait function in children with cerebral palsy yielded mixed results (Begnoche and Pitetti, 2007; Klamroth et al., 2019; Willoughby et al., 2009). Thus, the overall effect size needed to be estimated using metaanalysis, and the effect size $\geq 0.5$ was considered clinically significant (Chen et al., 2018). The overall effect size $(d=0.52)$ obtained in the present review suggested that treadmill training is a clinically effective intervention for gait function in children with cerebral palsy. In fact, task-oriented repetitive movement is considered crucial for motor learning in children with cerebral palsy (Nudo et al., 1996; Schindl et al., 2000). The reciprocal movement in the lower extremities during treadmill training likely has a positive effect on gait function in children with cerebral palsy.

Subgroup analyses yielded a large effect size for gait endurance in the present review $(d=0.85)$. The included studies investigated interventions with relatively long sessions, ranging from $15 \mathrm{~min}$ to $30 \mathrm{~min}$. A previous systematic review reported that treadmill training is an effective intervention for increasing cardiopulmonary function and strengthening the lower extremities in children with cerebral palsy (Maltais et al., 2014; Ruiz et al., 2009; Verschuren et al., 2013). Additionally, a systematic review and meta-analysis on treadmill training reported increased gait distance as an outcome in stroke patients. In particular, low-intensity continuous exercise has been shown to be effective for endurance of lower extremity muscles while avoiding the risk of joint injuries (Provost et al., 2007), which was indicated by the large effect size in the present meta-analysis.

The effect of treadmill training was significant with regard to limb support time, with a moderate effect size $(d=0.73)$. Children with cerebral palsy have weakness and spasticity of lower extremity (Cherng et al., 2007; Maltais et al., 2014). So many children with cerebral palsy have weight bearing related disability (Polese et al., 2013). Treadmill training includes partial body weight support or body weight support (Mehrholz et al., 2017; Molina-Rueda et al., 2010). In fact, weight bearing training is good for stability, bone mineral density and muscle strengthening of lower extremity (Hösl et al., 2018; Ruiz et al., 2009; Verschuren et al., 2013). Also, repetitive movement of joint during treadmill training decrease spasticity by dynamic muscle stretching and increase dorsiflexor muscle strengthening (Hösl et al., 2018). This is likely the reason for the clinically significant effect of treadmill training on limb support time in children with cerebral palsy in the present review.

The effect of treadmill training was significant with regard to gait speed, with a moderate effect size $(d=0.52)$. The clinically significant result suggests that repetitive gait training is effective 
for gait speed. In general, the gait pattern of cerebral palsy children is associated with the use of an inefficient kinetic mechanism due to cocontraction of proximal and distal muscles related to abnormal muscle tone (Hashiguchi et al., 2018). However, repetitive gait training using a treadmill promotes automatic and rhythmic gait in children with cerebral palsy by activating the central pattern generator of the spinal cord and improves coordination of agonistantagonist muscles in the lower extremities (Drew et al., 2008; Grecco et al., 2013). In addition, treadmill training is effective for strengthening the leg muscles, and it promotes muscle synergies in children with cerebral palsy, contributing to efficient movement (Drew et al., 2008; Mutlu et al., 2009). This is likely the reason for the clinically significant effect of treadmill training on gait speed in children with cerebral palsy in the present review (Polese et al., 2013), similar to a previous systematic review and metaanalysis that reported a clinically significant effect of treadmill training on gait speed in stroke patients.

The effects of training on cadence $(d=0.14)$ and step length $(d=$ $0.21)$ were small and statistically insignificant, unlike the effects on gait endurance, gait speed and limb support time. According to a previous study, cadence and step length are related to gait pattern and indicate segmental alignment (Chow et al., 2006). In individuals with a normal gait pattern, there is a positive correlation between cadence and step length (Chow et al., 2006; Morris et al., 1998). In fact, improvement of the pathological gait pattern in children with cerebral palsy requires interventions that are more specific than treadmill training, such as adjusting inadequate lever arms, strengthening stabilizer muscles, and controlling overpowered antagonist muscles (Gage, 1993; Schutte et al., 2000). Task-oriented training, including treadmill training, is limited with regard to improving motion kinetics by increasing compensatory movement (Massie et al., 2009). Therefore, repetitive gait training using the treadmill is likely inadequate for improving the gait pattern in children with cerebral palsy, but is effective for improving gait endurance and speed. However, considering that the effects on cadence and step length were estimated according to three studies and two studies, respectively, in this meta-analysis, caution is warranted when generalizing the results, and the effects need to be analyzed in additional studies in the future.

With regard to the quality of the included studies, the mean PEDro score for the studies was 6.25 , indicating good quality, especially considering that the average PEDro score is 5.0 according to the scale developer (Yamato et al., 2018). With regard to publication bias, the assessment with the duval and tweedie trim and fill method yielded the same correlation and observed values, sug- gesting low publication bias.

The present review has some limitations. It is difficult to generalize the results owing to the small number of included studies. Also, there are a lot of variables that indicate gait function. But this review not included many variables for gait function. Additionally, the review included relatively old studies, suggesting the need for a review of recent studies, especially those involving recently developed measures of gait function. Finally, future reviews should apply segmentation, with greater specificity, such as segmentation of the intervention period and type and duration of cerebral palsy.

The present systematic review and meta-analysis are regarding the effects of treadmill training on gait function in children with cerebral palsy. The effects of gait endurance, gait speed and limb support time were effective, but have clinically insufficient on cadence and step length. However, the observed significant effect of treadmill training on overall gait function suggests that it is an effective intervention to improve gait function in children with cerebral palsy. Therefore, the results of this review are expected to serve as important therapeutic evidence for the use of treadmill training as a community and clinical intervention in children with cerebral palsy.

\section{CONFLICT OF INTEREST}

No potential conflict of interest relevant to this article was reported.

\section{REFERENCES}

Begnoche DM, Pitetti KH. Effects of traditional treatment and partial body weight treadmill training on the motor skills of children with spastic cerebral palsy. A pilot study. Pediatr Phys Ther 2007;19:11-19.

Birgani PM, Ashtiyani M, Rasooli A, Shahrokhnia M, Shahrokhi A, Mirbagheri MM. Can an anti-gravity treadmill improve stability of children with cerebral palsy? Conf Proc IEEE Eng Med Biol Soc 2016;2016: 5465-5468.

Borenstein M, Hedges LV, Higgins JP, Rothstein HR. Introduction to meta-analysis. Chichester: John Wiley \& Sons; 2011.

Chen Y, Fanchiang HD, Howard A. Effectiveness of virtual reality in children with cerebral palsy: a systematic review and meta-analysis of randomized controlled trials. Phys Ther 2018;98:63-77.

Cherng RJ, Liu CF, Lau TW, Hong RB. Effect of treadmill training with body weight support on gait and gross motor function in children with spastic cerebral palsy. Am J Phys Med Rehabil 2007;86:548-555. 
Chow DH, Holmes AD, Lee CK, Sin SW. The effect of prosthesis alignment on the symmetry of gait in subjects with unilateral transtibial amputation. Prosthet Orthot Int 2006;30:114-128.

Chrysagis N, Skordilis EK, Stavrou N, Grammatopoulou E, Koutsouki D. The effect of treadmill training on gross motor function and walking speed in ambulatory adolescents with cerebral palsy: a randomized controlled trial. Am J Phys Med Rehabil 2012;91:747-760.

Dodd KJ, Foley S. Partial body-weight-supported treadmill training can improve walking in children with cerebral palsy: a clinical controlled trial. Dev Med Child Neurol 2007;49:101-105.

Drew T, Kalaska J, Krouchev N. Muscle synergies during locomotion in the cat: a model for motor cortex control. J Physiol 2008;586:1239-1245.

Gage JR. Gait analysis. An essential tool in the treatment of cerebral palsy. Clin Orthop Relat Res 1993;(288):126-134.

Gharib NM, El-Maksoud GM, Rezk-Allah SS. Efficacy of gait trainer as an adjunct to traditional physical therapy on walking performance in hemiparetic cerebral palsied children: a randomized controlled trial. Clin Rehabil 2011;25:924-934.

Grecco LA, Tomita SM, Christovão TC, Pasini H, Sampaio LM, Oliveira CS. Effect of treadmill gait training on static and functional balance in children with cerebral palsy: a randomized controlled trial. Braz J Phys Ther 2013;17:17-23.

Hashiguchi Y, Ohata K, Osako S, Kitatani R, Aga Y, Masaki M, Yamada S. Number of synergies is dependent on spasticity and gait kinetics in children with cerebral palsy. Pediatr Phys Ther 2018;30:34-38.

Hösl M, Böhm H, Eck J, Döderlein L, Arampatzis A. Effects of backwarddownhill treadmill training versus manual static plantarflexor stretching on muscle-joint pathology and function in children with spastic Cerebral Palsy. Gait Posture 2018;65:121-128.

Johnson CC. The benefits of physical activity for youth with developmental disabilities: a systematic review. Am J Health Promot 2009;23:157167.

Johnston TE, Watson KE, Ross SA, Gates PE, Gaughan JP, Lauer RT, Tucker CA, Engsberg JR. Effects of a supported speed treadmill training exercise program on impairment and function for children with cerebral palsy. Dev Med Child Neurol 2011;53:742-750.

Klamroth S, Gaßner H, Winkler J, Eskofier B, Klucken J, Pfeifer K, Steib S. Interindividual balance adaptations in response to perturbation treadmill training in persons with parkinson disease. J Neurol Phys Ther 2019;43:224-232.

Lepage C, Noreau L, Bernard PM. Association between characteristics of locomotion and accomplishment of life habits in children with cerebral palsy. Phys Ther 1998;78:458-469.

Macaskill P, Walter SD, Irwig L. A comparison of methods to detect publication bias in meta-analysis. Stat Med 2001;20:641-654.
Macko RF, Smith GV, Dobrovolny CL, Sorkin JD, Goldberg AP, Silver $\mathrm{KH}$. Treadmill training improves fitness reserve in chronic stroke patients. Arch Phys Med Rehabil 2001;82:879-884.

Maltais DB, Wiart L, Fowler E, Verschuren O, Damiano DL. Health-related physical fitness for children with cerebral palsy. J Child Neurol 2014; 29:1091-1100.

Massie C, Malcolm MP, Greene D, Thaut M. The effects of constraint-induced therapy on kinematic outcomes and compensatory movement patterns: an exploratory study. Arch Phys Med Rehabil 2009;90:571579.

Mehrholz J, Harvey LA, Thomas S, Elsner B. Is body-weight-supported treadmill training or robotic-assisted gait training superior to overground gait training and other forms of physiotherapy in people with spinal cord injury? A systematic review. Spinal Cord 2017;55:722-729.

Moher D, Shamseer L, Clarke M, Ghersi D, Liberati A, Petticrew M, Shekelle P, Stewart LA; PRISMA-P Group. Preferred reporting items for systematic review and meta-analysis protocols (PRISMA-P) 2015 statement. Syst Rev 2015;4:1.

Molina-Rueda F, Aguila-Maturana AM, Molina-Rueda MJ, MiangolarraPage JC. Treadmill training with or without partial body weight support in children with cerebral palsy: systematic review and meta-analysis. Rev Neurol 2010;51:135-145.

Morris M, Iansek R, Matyas T, Summers J. Abnormalities in the stride length-cadence relation in parkinsonian gait. Mov Disord 1998;13:6169.

Mutlu A, Krosschell K, Spira DG. Treadmill training with partial bodyweight support in children with cerebral palsy: a systematic review. Dev Med Child Neurol 2009;51:268-275.

Nicholson K, Lennon N, Church C, Miller F. Gait analysis parameters and walking activity pre- and postoperatively in children with cerebral palsy. Pediatr Phys Ther 2018;30:203-207.

Nudo RJ, Wise BM, SiFuentes F, Milliken GW. Neural substrates for the effects of rehabilitative training on motor recovery after ischemic infarct. Science 1996;272:1791-1794.

Polese JC, Ada L, Dean CM, Nascimento LR, Teixeira-Salmela LF. Treadmill training is effective for ambulatory adults with stroke: a systematic review. J Physiother 2013;59:73-80.

Provost B, Dieruf K, Burtner PA, Phillips JP, Bernitsky-Beddingfield A, Sullivan KJ, Bowen CA, Toser L. Endurance and gait in children with cerebral palsy after intensive body weight-supported treadmill training. Pediatr Phys Ther 2007;19:2-10.

Ruiz JR, Castro-Piñero J, Artero EG, Ortega FB, Sjöström M, Suni J, Castillo MJ. Predictive validity of health-related fitness in youth: a systematic review. Br J Sports Med 2009;43:909-923.

Saquetto M, Carvalho V, Silva C, Conceição C, Gomes-Neto M. The ef- 
fects of whole body vibration on mobility and balance in children with cerebral palsy: a systematic review with meta-analysis. J Musculoskelet Neuronal Interact 2015;15:137-144.

Schalock RL, Gomez LE, Verdugo MA, Claes C. Evidence and evidencebased practices: are we there yet? Intellect Dev Disabil 2017;55:112-119.

Schiariti V, Selb M, Cieza A, O’Donnell M. International Classification of Functioning, Disability and Health Core Sets for children and youth with cerebral palsy: a consensus meeting. Dev Med Child Neurol 2015; 57:149-158.

Schindl MR, Forstner C, Kern H, Hesse S. Treadmill training with partial body weight support in nonambulatory patients with cerebral palsy. Arch Phys Med Rehabil 2000;81:301-306.

Schutte LM, Narayanan U, Stout JL, Selber P, Gage JR, Schwartz MH. An index for quantifying deviations from normal gait. Gait Posture 2000; 11:25-31.

Schwartz MH, Rozumalski A. The Gait Deviation Index: a new comprehensive index of gait pathology. Gait Posture 2008;28:351-357.
Verschuren O, Maltais DB, Douma-van Riet D, Kruitwagen C, Ketelaar M. Anaerobic performance in children with cerebral palsy compared to children with typical development. Pediatr Phys Ther 2013;25:409-413.

Willoughby KL, Dodd KJ, Shields N. A systematic review of the effectiveness of treadmill training for children with cerebral palsy. Disabil Rehabil 2009;31:1971-1979.

Willoughby KL, Dodd KJ, Shields N, Foley S. Efficacy of partial body weight-supported treadmill training compared with overground walking practice for children with cerebral palsy: a randomized controlled trial. Arch Phys Med Rehabil 2010;91:333-339.

Yamato TP, Arora M, Stevens ML, Elkins MR, Moseley AM. Quality, language, subdiscipline and promotion were associated with article accesses on Physiotherapy Evidence Database (PEDro). Physiotherapy 2018;104:122-128.

Zwicker JG, Mayson TA. Effectiveness of treadmill training in children with motor impairments: an overview of systematic reviews. Pediatr Phys Ther 2010;22:361-377. 